\title{
Electronic structure and crystal phase stability of palladium hydrides
}

\author{
Abdesalem Houari* \\ Theoretical Physics Laboratory, Department of Physics, University of Bejaia, Bejaia, Algeria \\ Samir F. Matar \\ CNRS, ICMCB, Université de Bordeaux, 33600 Pessac, France \\ Volker Eyert田 \\ Materials Design SARL, 92120 Montrouge, France
}

(Dated: November 4, 2014)

\begin{abstract}
The results of electronic structure calculations for a variety of palladium hydrides are presented. The calculations are based on density functional theory and used different local and semilocal approximations. The thermodynamic stability of all structures as well as the electronic and chemical bonding properties are addressed. For the monohydride, taking into account the zero-point energy is important to identify the octahedral $\mathrm{Pd}-\mathrm{H}$ arrangement with its larger voids and, hence, softer hydrogen vibrational modes as favorable over the tetrahedral arrangement as found in the zincblende and wurtzite structures. Stabilization of the rocksalt structure is due to strong bonding of the $4 d$ and $1 s$ orbitals, which form a characteristic split-off band separated from the main $d$-band group. Increased filling of the formerly pure $d$ states of the metal causes strong reduction of the density of states at the Fermi energy, which undermines possible long-range ferromagnetic order otherwise favored by strong magnetovolume effects. For the dihydride, octahedral $\mathrm{Pd}-\mathrm{H}$ arrangement as realized e.g. in the pyrite structure turns out to be unstable against tetrahedral arrangemnt as found in the fluorite structure. Yet, from both heat of formation and chemical bonding considerations the dihydride turns out to be less favorable than the monohydride. Finally, the vacancy ordered defect phase $\mathrm{Pd}_{3} \mathrm{H}_{4}$ follows the general trend of favoring the octahedral arrangement of the rocksalt structure for $\mathrm{Pd}: \mathrm{H}$ ratios less or equal to one.
\end{abstract}

PACS numbers: 61.50.Lt, 71.20.-b, 75.50.Cc, 88.30.rd

Keywords: Palladium hydride, electronic structure, phase stability, magnetism

\section{INTRODUCTION}

Metal hydrides have attracted a lot of attention in the last decades due to a variety of exciting applications especially with regard to the environmentally friendly storage and use of energy. In this context, they have proven as very promising candidates for hydrogen storage, fuel cells applications 1,2 , and heat storage for the solar-energy industry ${ }^{3}$. These auspicious perspectives are complemented by strong interest from the point of view of fundamental research inter alia because of their role as possible candidates for high-temperature superconductivity ${ }^{4}$. Since hydrogen insertion can act as negative pressure it may also cause a switching between different electronic or magnetic states, as has been found, e.g., in intermetallic cerium compounds ${ }^{5}$.

Especially the palladium-hydrogen system has been investigated since long6.7 due to the fact that palladium allows to absorb comparatively large amounts of hydrogen ${ }^{8-16}$. For this reason, much interest has centered about the maximum amount of hydrogen to be absorbed in the metal as well as the possible hydrogen positions in the parent structure. This has initiated several structural characterizations as well as theoretical studies. Many investigations focused on the question, which open voids in the underlying face-centered cubic lattice of palladium metal are most likely to be occupied by hydrogen. Especially, for the monohydride the exact position of the hydrogen atoms was a matter of long dispute. According to neutron diffraction data, hydrogen atoms occupy the octahedral interstices of the face-centered cubic lattice of $\mathrm{Pd}$ leading to the rocksalt structure $13,14,16,17$. However, depending on temperature a transfer of substantial amounts of hydrogen to the tetrahedral interstices, leading to the zincblende structure, has been inferred from neutron diffraction data 18 . For the palladium-deuterium system a partial occupation of the tetrahedral site was also reported and attributed to the lower zero-point energy of the D interstitial as compared to $\mathrm{H}^{19}$.

Due to their increased hydrogen content as compared to the monohydrides, transition metal dihydrides have also come into focus quite early, see, e.g. the work by Switendick and references therein ${ }^{20,21}$. In this case, there was agreement on the fluorite structure. More recently, the formation of superabundant vacancy structures was observed at high temperatures and high hydrogen pressures, possibly the most prominent example being $\mathrm{Pd}_{3} \mathrm{H}_{4}$, which arises from the monohydride on removing $\mathrm{Pd}$ and forms an ordered structure ${ }^{22-24}$. This vacancy formation is accompanied by a compression of the lattice.

Due to the high interest in the palladium-hydrogen system, a number of theoretical studies have been performed ranging from simplified non-self-consistent calculations using atomic potentials $20,21,25,26$ to fully selfconsistent relativistic state-of-the-art electronic structure 
calculations as based on density functional theory and (semi-)local approximations to the exchange-correlation functional ${ }^{27-33}$. Nevertheless, even the early work by Switendick revealed considerable distortions of the $\mathrm{Pd}$ band structure on hydrogenation and allowed to rule out previous rigid-band considerations ${ }^{20,21}$. In particular, based on his finding of only a partial charge transfer from hydrogen to the metal, Switendick was the first to abandon the then popular anion and proton models, which were based on an ionic picture. These findings were lateron confirmed by more refined calculations ${ }^{25-27,29}$. More recent theoretical work centered about the issue of the hydrogen site in $\mathrm{PdH}$, which was motivated by the afore mentioned disputes $31-33$.

As is well known from a number of studies, hydrogen insertion in metals may induce long-range magnetic order via strong magnetovolume effects. This is especially relevant for paramagnetic palladium, which is at the verge of a ferromagnetic instability. Yet, in general there is a strong competition between the magnetovolume effects enhancing magnetization and the effects of hydrogen bonding to the underlying metal substructure leading to the suppression of the magnetic order ${ }^{34.35}$.

The present work employed electronic structure calculations as based on density functional theory and focusses on the structure-property relationships of several palladium hydrides. We thus follow two main goals: First, emphasis is on the thermodynamic stability of a variety of palladium hydrides with perfect stoichiometry and different crystal structures. This includes three possible structures of the monohydride $\mathrm{PdH}$ : the observed rocksalt structure, the zincblende structure, and the hexagonal wurtzite structure. In addition, the hydrogen overstoichiometric palladium dihydride $\mathrm{PdH}_{2}$ is considered in both the fluorite and the related pyrite structure. Finally, the above mentioned recent experiments suggested to investigate the new ordered $\mathrm{Pd}$-defect phase $\mathrm{Pd}_{3} \mathrm{H}_{4}$. The second goal of this work centers about the calculation of the electronic and magnetic properties as well as chemical bonding indicators, which lead to a deeper understanding of the various phases.

\section{COMPUTATIONAL METHODS}

The first principles calculations were based on density functional theory 36,37 with exchange-correlation effects accounted for by both the local and semilocal approximation. Two complementary tools were employed:

In a first step, the Vienna ab-initio simulation program (VASP) as implemented in the MedeA ${ }^{\circledR}$ computational environment of Materials Design was used to perform total-energy and force calculations aiming at an optimization of the structures 38,39 . The exchange-correlation functional was considered at three different levels, namely, within the local density approximation ${ }^{40}$, the generalized gradient approximation (GGA) by Perdew, Burke, and Ernzerhof (PBE) $\stackrel{41}{ }$, and the PBEsol scheme recently devised especially for the improved treatment of solids 42 . The single-particle equations were solved using the projector-augmented wave (PAW) method ${ }^{43,44}$ with a plane-wave basis with a cutoff of $326.203 \mathrm{eV}$. The Brillouin zone was sampled using a Monkhorst-Pack mesh with a spacing below $0.1 \AA^{-1}$ leading to $27 \times 27 \times 27 \mathbf{k}$ points for the rocksalt and zincblende structures as well as $26 \times 26 \times 13 \mathrm{k}$-points for the wurtzite structure 45 . Furthermore, $25 \times 25 \times 25$ and $16 \times 16 \times 16 \mathrm{k}$-points were used for the dihydride and $\mathrm{Pd}_{3} \mathrm{H}_{4}$, respectively. Phonon spectra were calculated using the Phonon software $\frac{46}{}$, which is likewise part of the MedeA ${ }^{\circledR}$ computational environment and used the forces evaluated with the VASP code.

Once the optimized structures were known, calculations of the electronic structure and the chemical bonding properties were carried out using the full-potential augmented spherical wave (ASW) method in its scalarrelativistic implementation 47,48 . Exchange and correlation effects were considered using the GGA parametrization of $\mathrm{Wu}$ and Cohen 49 . In the ASW method, the wave function is expanded in atom-centered augmented spherical waves, which are Hankel functions and numerical solutions of Schrödinger's equation, respectively, outside and inside the so-called augmentation spheres. In order to optimize the basis set and enhance the variational freedom, additional augmented spherical waves were placed at carefully selected interstitial sites. The choice of these sites as well as the augmentation radii were automatically determined using the sphere-geometry optimization algorithm ${ }^{50}$. Self-consistency was achieved by a highly efficient algorithm for convergence acceleration 51 until the variation of the atomic charges was smaller than $10^{-8}$ electrons and the variation of the total energy was smaller than $10^{-8}$ Ryd. Brillouin zone integrations were performed using the linear tetrahedron method 52 with $27 \times 27 \times 27 \mathbf{k}$-points for face-centered cubic Pd, $26 \times 26 \times 26 \mathbf{k}$-points for the rocksalt structure, $25 \times 25 \times 25$ k-points for the zincblende structure, and $24 \times 24 \times 12 \mathrm{k}$ points for the wurtzite structure. In addition, $24 \times 24 \times 24$ and $15 \times 15 \times 15 \mathrm{k}$-points were used for the dihydride and $\mathrm{Pd}_{3} \mathrm{H}_{4}$, respectively.

In the present work, a new full-potential version of the ASW method was employed ${ }^{48}$. In this version, the electron density and related quantities are given by spherical harmonics expansions inside the muffin-tin spheres while, in the remaining interstitial region, a representation in terms of atom-centered Hankel functions is used 53 . However, in contrast to previous related implementations, no so-called multiple- $\kappa$ basis set is needed, rendering the method computationally nearly as efficient as the original ASW scheme. 


\section{RESULTS}

\section{A. Monohydride $\mathrm{PdH}$}

As mentioned above, three different structures were considered for the monohydride, namely, the rocksalt structure with hydrogen placed at the octahedral sites of the face-centered cubic lattice formed by elemental $\mathrm{Pd}$, the zincblende structure with hydrogen being at the tetrahedral sites, and the hexagonal wurtzite structure, which also has the hydrogen atoms at the center of the tetrahedral voids of the Pd sublattice. All structures were fully optimized, i.e. the lattice vectors as well as the internal coordinates were relaxed using VASP. The results are summarized in Table \, which gives calculated

TABLE I: Calculated equilibrium properties of $\mathrm{PdH}$ in the rocksalt (RS), zincblende (ZB), and wurtzite (WZ) structures: total energies $E$ per formula unit, lattice constants $a$ and $c$, as well as hydrogen parameters $u$ within LDA/PBEsol/PBE. Calculated properties of palladium metal and experimental data (from Refs. 54, 55, and 14) as well as previous calculational results are added for comparison.

\begin{tabular}{|c|c|c|c|c|c|c|c|}
\hline \multirow{7}{*}{$\mathrm{Pd}$} & & \multicolumn{3}{|c|}{$E-E_{R S}(\mathrm{meV})$} & \multicolumn{3}{|c|}{$a(\AA)[, c, u]$} \\
\hline & \multirow{6}{*}{$\begin{array}{l}\text { this work } \\
\text { Ref. } 28 \\
\text { Ref. } 30 \\
\text { Ref. } 31 \\
\text { Ref. } 33 \\
\text { Ref. } 54\end{array}$} & & & & 3.84 & 3.87 & 3.94 \\
\hline & & & & & 3.87 & & \\
\hline & & & & & 3.88 & & \\
\hline & & & & & & & 3.97 \\
\hline & & & & & 3.88 & & \\
\hline & & & & & & 3.89 & \\
\hline \multirow[t]{8}{*}{$\mathrm{RS}$} & this work & \multirow{8}{*}{\multicolumn{3}{|c|}{0}} & 4.03 & 4.06 & 4.12 \\
\hline & Ref. 28 & & & & 4.12 & & \\
\hline & Ref. 56 & & & & 4.08 & & \\
\hline & Ref. 30 & & & & 4.07 & & \\
\hline & Ref. 31 & & & & & & 4.15 \\
\hline & Ref. 33 & & & & 4.06 & & \\
\hline & Ref. 55 & & & & & 4.09 & \\
\hline & Ref. 14 & & & & & 4.07 & \\
\hline \multirow[t]{3}{*}{$\mathrm{ZB}$} & this work & \multirow[t]{3}{*}{-17} & \multirow[t]{3}{*}{-25} & \multirow[t]{3}{*}{-83} & 4.14 & 4.17 & 4.23 \\
\hline & Ref. 30 & & & & 4.17 & & \\
\hline & Ref. 31 & & & & & & 4.26 \\
\hline \multirow[t]{3}{*}{ WZ } & \multirow[t]{3}{*}{ this work } & \multirow[t]{3}{*}{+17} & \multirow[t]{3}{*}{+9} & \multirow[t]{3}{*}{-49} & 2.88 & 2.90 & 2.95 \\
\hline & & & & & 4.93 & 4.97 & 5.02 \\
\hline & & & & & 0.36 & 0.36 & 0.36 \\
\hline
\end{tabular}

lattice parameters and internal parameters (in case of the wurtzite structure) arising from the three exchangecorrelation functionals mentioned above together with available theoretical and experimental data from the literature. In all cases, the agreement with previous structural data is very good. This is true also for elemental $\mathrm{Pd}$, which is considered in the upper part of the table for comparison. Especially noteworthy are the experimental results of Refs. $\underline{54}, \underline{55}$, and 14 , which reveal very good agreement with the calculations using the PBEsol functional.

Table \also lists the calculated total energies, which are given relative to those of the rocksalt structure, again for all three exchange-correlation functionals. Obviously, the zincblende structure is found to be most stable for all three functionals. Yet, this is in contrast to most experimental findings $13,14,16,17$ despite some hints at an occupation of the tetrahedral site $\stackrel{18,19}{ }$. In addition, it is at variance with the situation of hydrogen in nickel, where calculations reveal the octahedral site as the most stable one ${ }^{57}$.

In order to resolve the issue, we have complemented the calculations underlying Table @ by calculations of the phonon spectra of all three structures, thereby focusing on the use of the PBEsol exchange-correlation functional, as this gives the best structural data in comparison with experiment. From these calculations, zero-point energies of 94,219 , and $214 \mathrm{meV}$ (per formula unit) were obtained for the rocksalt, zincblende, and wurtzite structure, respectively. Obviously, addition of the zero-point energies reverses the order of total energies found for the three different structures, leaving the rocksalt structure as the most stable one. Specifically, with the zero-point energies included, the zincblende and wurtzite structures have energies, which are 100 and $129 \mathrm{meV}$, respectively, above that of the rocksalt structure.

The above striking differences can be understood from a closer inspection of the phonon dispersions calculated for both the rocksalt and the zincblende structure as displayed in Fig. 1. Results for the wurtzite structure are very similar to those obtained for the zincblende structure. For the cubic structures the phonon dispersions fall into two groups with three phonon branches each. While the lower set of branches extends up to about $7 \mathrm{THz}$ for both structures, strong differences are found for the upper group. Specifically, these branches are found at frequencies around $32 \mathrm{THz}$ for the zincblende structure, whereas for the rocksalt structure they extend from about $5 \mathrm{THz}$ to a maximum of about $22 \mathrm{THz}$, thus explaining the much higher zero-point energy of the former structure.

As a deeper analysis reveals, the lower group of branches corresponds to phonon modes with a cooperative motion of $\mathrm{Pd}$ and $\mathrm{H}$, whereas the higher lying branches exclusively involve motion of the rather rigid $\mathrm{H}$ and $\mathrm{Pd}$ sublattices relative to each other and, hence, strongly affect the $\mathrm{Pd}-\mathrm{H}$ bonds. In this context it is interesting to relate the differences in zero-point energy to the differences in $\mathrm{Pd}-\mathrm{H}$ bond lengths in all three structures. We find $2.03 \AA, 1.80 \AA$ Aand 1.79/1.81 $\AA$, respectively, for the rocksalt, zincblende, and wurtzite structure (with the first value given for the latter structure referring to the bond parallel to the hexagonal $c$ axis). These bond length are much larger than the sum of the covalent radii $(1.28$ Aand $0.32 \AA$ ) due to the fact that the lattice spacing is governed by the Pd-Pd bonds. As a consequence, hydrogen vibrations relative to the neighbouring $\mathrm{Pd}$ atoms 

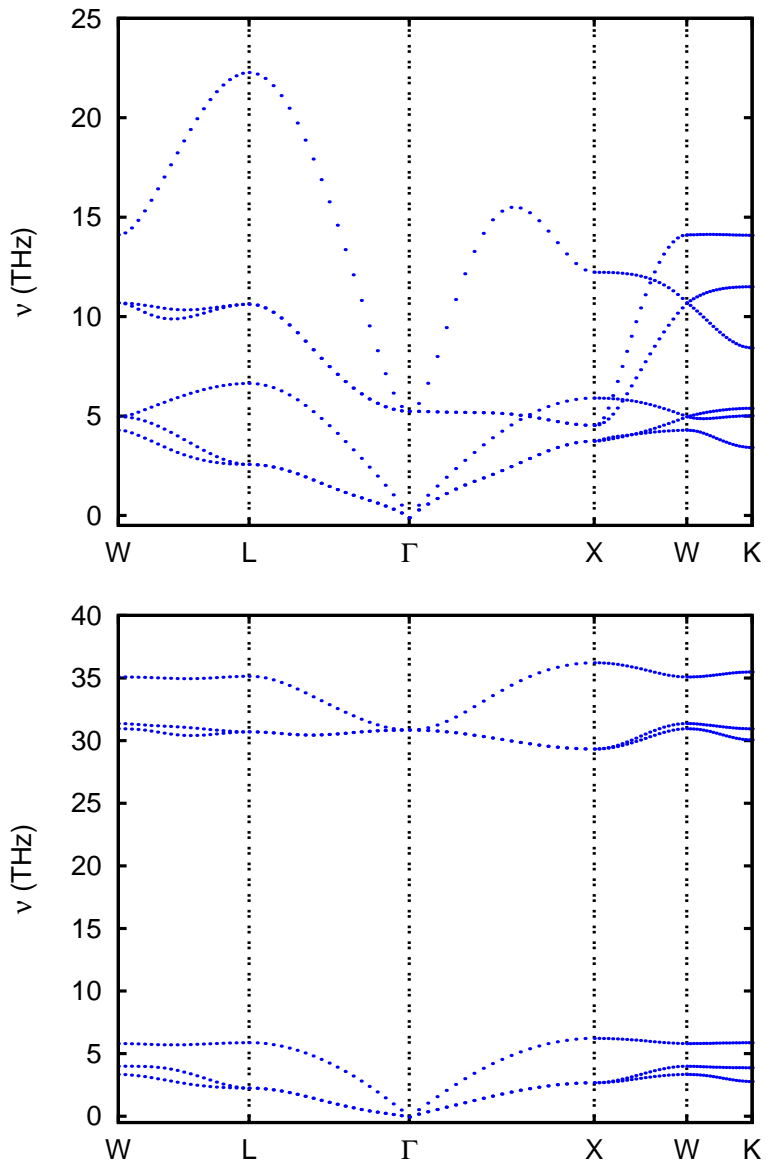

FIG. 1: Phonon dispersions of $\mathrm{PdH}$ in the rocksalt $(\mathrm{H}$ in octahedral site, top) and zincblende ( $\mathrm{H}$ in tetrahedral site, bottom) structure.

affect the metal-hydrogen bonds to a much lesser degree in the rocksalt structure as compared to the zincblende structure and are thus found at lower energies. This explains the strong difference in zero-point energies, which are roughly inversely proportional to the difference between the bond lengths and sum of the covalent radii.

In order to discuss the previous results from the electronic structure, calculations of the (partial) densities of states were performed using the ASW method. The results for elemental $\mathrm{Pd}$ as well as $\mathrm{PdH}$ in the rocksalt and zincblende structures are shown in Fig. 2, For all three systems we find dominant contributions from the $\mathrm{Pd} 4 d$ states, which are complemented by small admixtures from the $5 s p$ states especially in the low energy part of the spectrum. Two findings are especially noteworthy: First, the main body of the $\mathrm{Pd} 4 d$ states is much narrower in the hydride structures as compared to elemental Pd. Second, for the hydrides a split-off band is observed at about $-8 \mathrm{eV}$ in very good agreement with photoemission data $5 \underline{-60}$. This band arises from almost equal contributions of the $\mathrm{Pd} 4 d$ and $\mathrm{H} 1 s$ states, which are almost identical in shape. The latter fact is a signa-
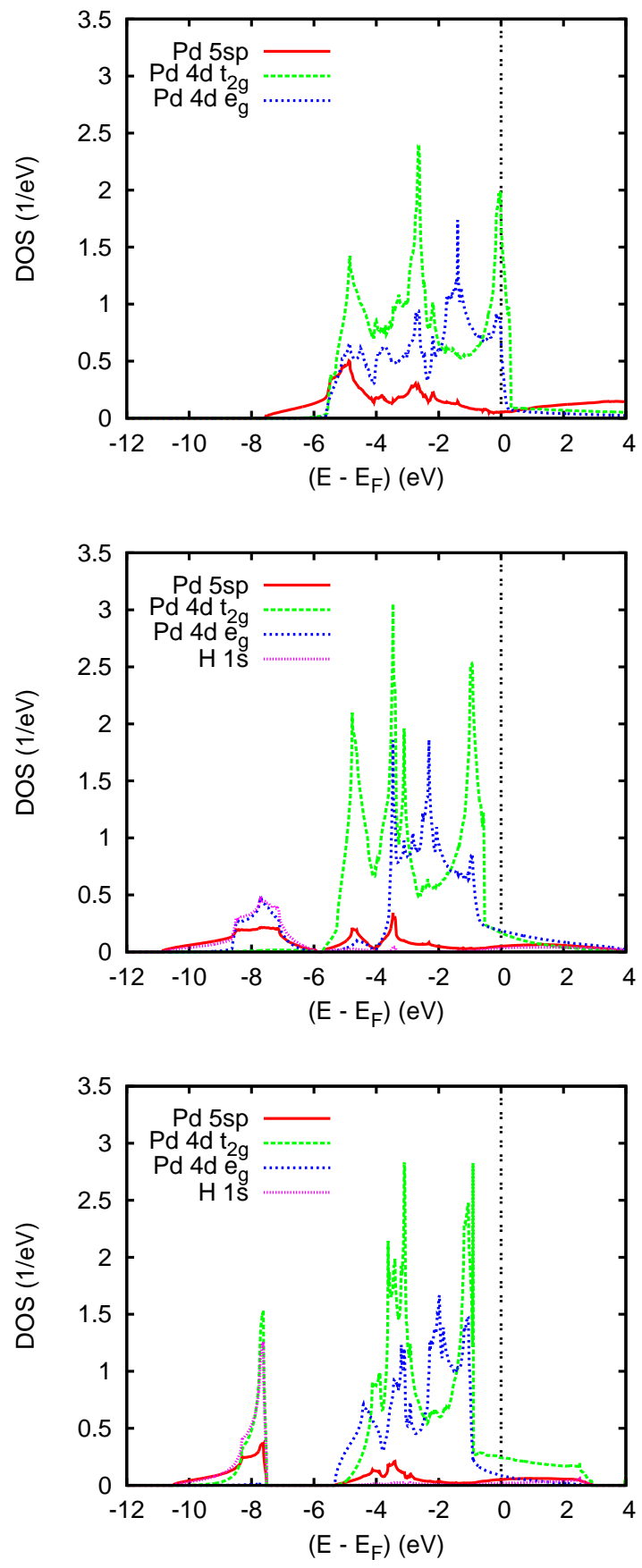

FIG. 2: Partial densities of states of Pd (top) and PdH in the rocksalt ( $\mathrm{H}$ in octahedral site, middle) and zincblende $(\mathrm{H}$ in tetrahedral site, bottom) structure.

ture of a strong overlap of these states. Indeed, as Fig. 2 reveals for the rocksalt structure, the split-off band comprises only those $4 d$ states, which form strong $\sigma$-type bonds with the $1 s$ states. In the octahedral arrangement of the rocksalt structure these are the $e_{g}$ states. In contrast, in the zincblende and wurtzite structures the palladium atoms are tetrahedrally coordinated and, hence, 
the strong $\sigma$-type $4 d$ - $1 s$ overlap is mediated by the $t_{2 g}$ orbitals. This leads to the characteristic shape of the splitoff band, which is similar for the zincblende and wurtzite structures but differs from that obtained for the rocksalt structure. Nevertheless, the strong $\sigma$-type overlap with the almost identical shape of the $\mathrm{Pd} 4 d$ and $\mathrm{H} 1 s$ contributions impressively confirms the predominantly covalent metal-hydrogen bonding of palladium hydride as put forward already by Switendick 20.21 . It explains the fact that the $\mathrm{Pd}-\mathrm{H}$ phases are rather metastable inasmuch as they readily decompose, contrary to very stable ionic hydrides, whose archetype is $\mathrm{MgH}_{2}$, where the electron transfer is much larger 61 . Interestingly, in cubic $\beta-\mathrm{MgPd}_{3} \mathrm{H}_{0.7}$ lowlying $\mathrm{Pd}-\mathrm{H}$ bonding states are also found. Yet, there are no noticable contributions from $\mathrm{Mg}$ in this energy region, which fact reveals the strong Pd-preference of hydrogen as well as the tendency to form strong covalent $\mathrm{Pd}-\mathrm{H}$ bonds $\frac{62}{}$.

The results obtained for rocksalt structure $\mathrm{PdH}$ are very similar to those shown by previous authors $\underline{26}-29$. In particular, Williams, Kübler, and Gelatt attribute the split-off band to the attractive potential arising from the interstitial hydrogen proton, which leads to strong overlap of the $4 d$ and $1 s$ states and thus lays ground for the chemical bonding of the hydrides. They find a very similar behavior as well for $\mathrm{NiH}$ and the neighboring hydrides in the $3 d$ and $4 d$ series.

It is interesting to compare the band structures of elemental $\mathrm{Pd}$ and $\mathrm{PdH}$ in the rocksalt structure, which are displayed in Fig. 3. They clearly reveal the downshift of the lowest band of $\mathrm{Pd}$ metal by about $2-3 \mathrm{eV}$ in good agreement with all previous calculations. However, while the early non-self-consistent calculations find this band completely separated from the higher lying bands $20,21,26$, we find a band crossing close to the $\mathrm{L}$ point in our calculation in accordance with previous self-consistent calculations ${ }^{29}$. The low-lying band at L seems to disperse up to $-4 \mathrm{eV}$ at the $\mathrm{W}$ point. However, the dominant $\mathrm{H}$ $1 s$ character, which is indicated in Fig. 3. always remains with the lowest band.

The downshift of the $d$ band group is most obvious from the (almost) dispersionless highest $d$ bands along the lines W-L and X-W-K, which straddle the Fermi energy in $\mathrm{Pd}$ but are found completely occupied in $\mathrm{PdH}$. As a result, as has been also mentioned by Gupta and Freeman $\stackrel{26}{ }$, the Fermi surface becomes much clearer and assumes the shape known from the nobel metals. Another consequence of this lowering is the drastic reduction of the density of states at the Fermi level from 2.47 states/eV to 0.46 states/eV in perfect agreement with previous calculations $20,21,29$.

We complement the above considerations with a discussion of the chemical bonding properties as they are provided by the crystal orbital overlap population (COOP) or the more recent covalence energy scheme (ECOV) used here 63.64 . Calculated results for the latter

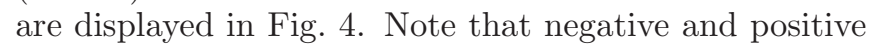
contributions to the covalence energy point to bonding
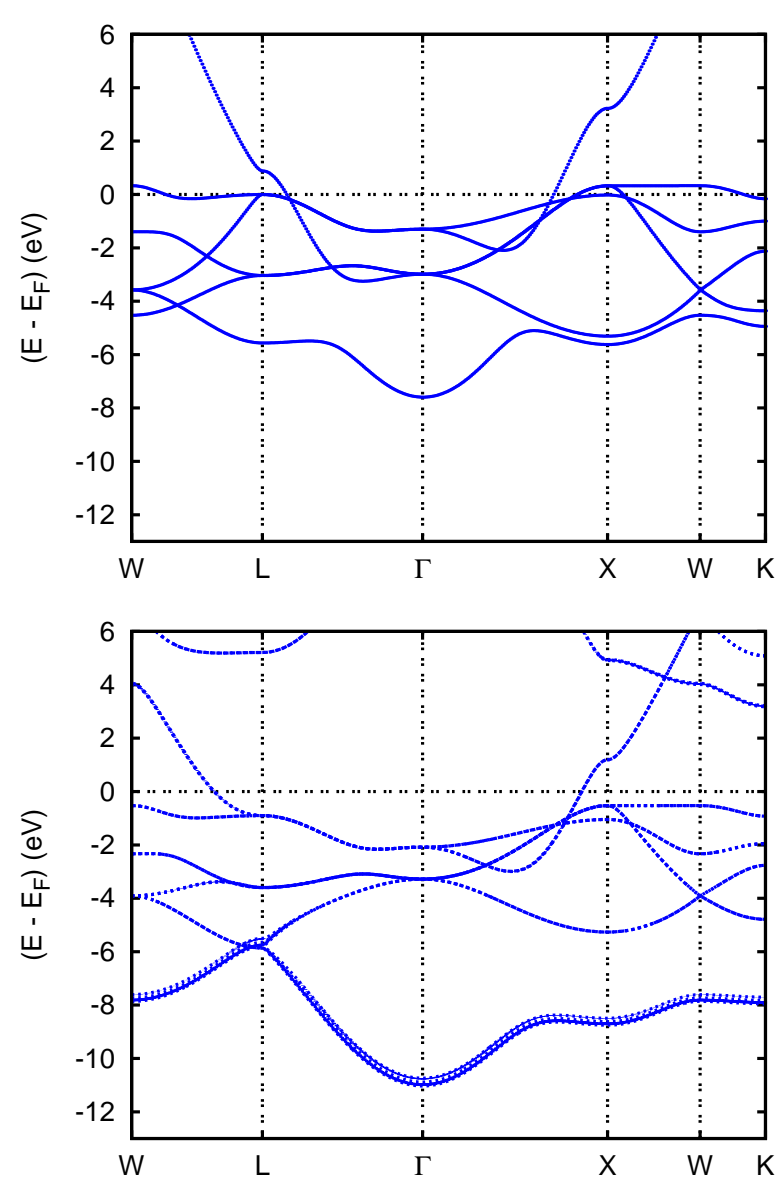

FIG. 3: Electronic bands of $\mathrm{Pd}$ (top) and $\mathrm{PdH}$ in the rocksalt ( $\mathrm{H}$ in octahedral site, bottom) structure. The width of the bars given with each band is a measure of the $\mathrm{H} 1 s$ contribution to the respective wave function.

and antibonding orbitals, respectively. Note also that by definition the covalence energy is a dimensionless quantity. For elemental $\mathrm{Pd}, d-d$ bonding and antibonding contributions found in the lower and upper range of the $4 d$ band, respectively, nearly outweigh each other. In contrast, $s p$ - $d$ bonding contributions extend up to about $-2 \mathrm{eV}$ and thus lead to a stabilization of the metal. These essential findings are recognized also in the hydride structures. However, due to the upshift of the Fermi level, the respective antibonding states attain a higher occupation and would destabilize the metallic framework were it not for the hydrogen bonding. While the overlap of the $\mathrm{Pd}$ $5 s p$ states with the hydrogen orbitals plays only a negligible role, the $4 d-1 s$ overlap as expected turns out to be essential for the stability of the hydride. Obviously, the split-off band comprises the bonding contributions, whereas the antibonding parts set in only at much higher energies and start to counterbalance the bonding states just above the Fermi energy.

As well known, transition-metal hydrides may show a tendency towards long-range magnetic order due to their expanded volumes 34,35 . In particular, previous cal- 

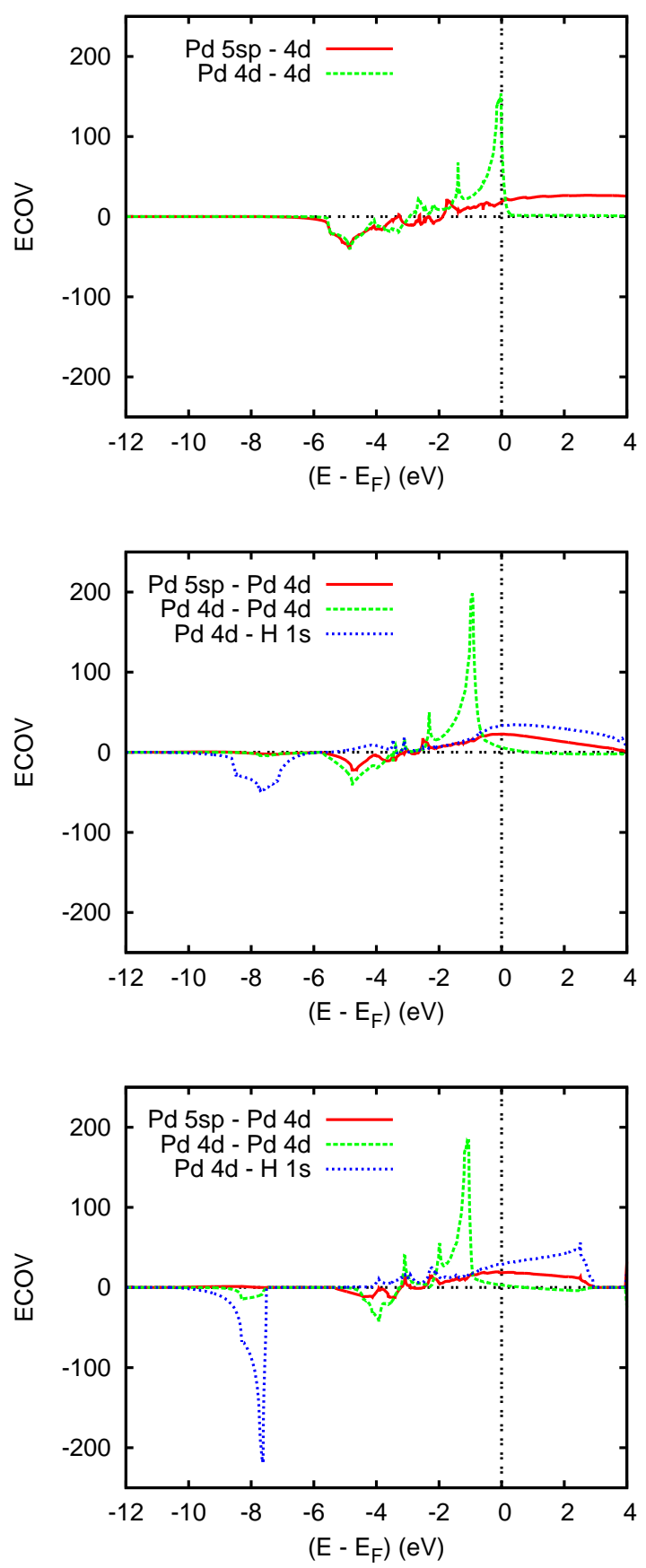

FIG. 4: Partial covalence energies of $\mathrm{Pd}$ (top) and $\mathrm{PdH}$ in the rocksalt ( $\mathrm{H}$ in octahedral site, middle) and zincblende $(\mathrm{H}$ in tetrahedral site, bottom) structure.

culations for elemental $\mathrm{Pd}$ were interpreted in terms of a possible ferromagnetic instability at expanded lattice constants ${ }^{65,66}$. For this reason, we performed additional spin-polarized calculations in order to check for stable ferromagnetic order. The results of spin-polarized ASW calculations are displayed in Fig. (5. The corresponding lowering of the total energy on including spin-polarization

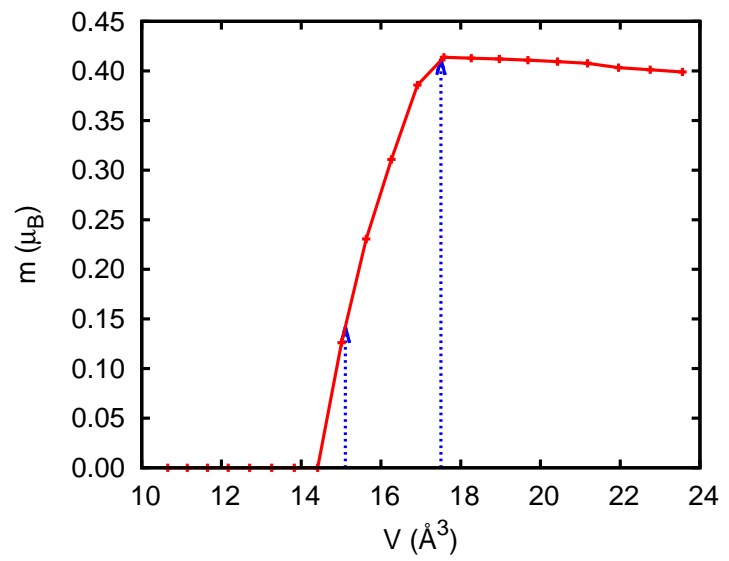

FIG. 5: Magnetic moment of Pd per unit cell as a function of volume. Connecting lines serve as guide to the eye only; arrows at $15.1 \AA^{3}$ and $17.5 \AA^{3}$ mark the equilibrium volumes of $\mathrm{Pd}$ and rocksalt $\mathrm{PdH}$, respectively.

is captured in Fig. 6. According to these findings, $\mathrm{Pd}$

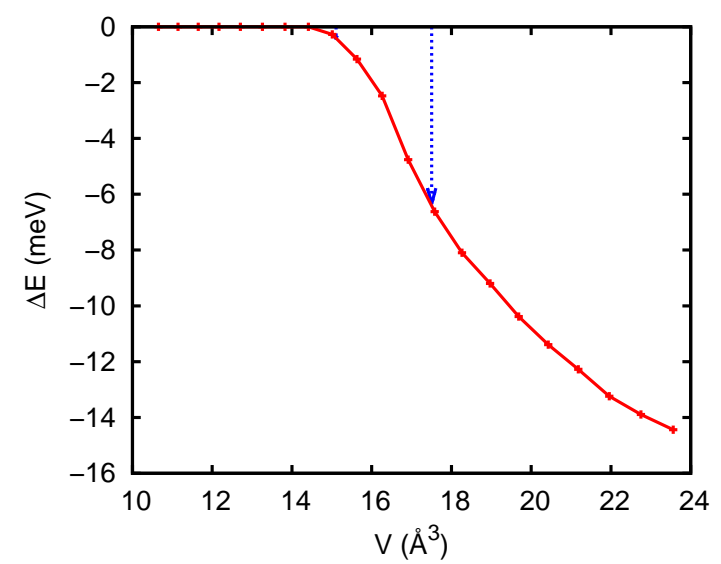

FIG. 6: Total energy difference between spin-polarized and spin-degenerate calculations of $\mathrm{Pd}$ as a function of volume. Connecting lines serve as guide to the eye only; arrows at $15.1 \AA^{3}$ and $17.5 \AA^{3}$ mark the equilibrium volumes of $\mathrm{Pd}$ and rocksalt $\mathrm{PdH}$, respectively.

metal is indeed on the verge of developing a finite magnetization; at the equilibrium lattice constant, it amounts to $\approx 0.14 \mu_{B}$. On expanding the lattice, the magnetization sharply increases until it reaches its saturation value of about $0.4 \mu_{B}$ at the lattice constant of $\mathrm{PdH}$. These findings may be easily understood from the strong peak in the density of states at the Fermi energy as seen in Fig. 2 which according to Stoner theory may cause a ferromagnetic instability. On expanding the lattice this peak narrows and becomes stronger, which fact is in favor of the energy gain due to magnetic ordering as deduced from the Stoner picture. Hence, were it only for the volume expansion effects brought by hydrogen in- 
sertion, PdH would develop stable ferromagnetic order. However, as discussed in connection with the densities of states shown in Fig. 2, hydrogen insertion, in addition to expanding the lattice, pushes the Fermi energy to the energy range above the strong $4 d$ peaks where the density of states is small. As a consequence, the magnetic instability is suppressed. From a different point of view, this suppression of magnetic ordering may be assigned to the quenching of the local magnetic moments by the spin pairing coming with the $\mathrm{Pd}-\mathrm{H}$ bonding. Given the densities of states of $\mathrm{Pd}$ metal shown in Fig. 2 this result is very likely to hold even at a reduced hydrogen content. Nevertheless, it is important to note that the energy gain on turning on spin-polarization and expanding the lattice is of the order of $10 \mathrm{meV}$ and, hence, any long-range ordering would occur at very low temperatures only.

\section{B. Dihydride $\mathrm{PdH}_{2}$}

Following the early work by Switendick, we extended the present study to the dihydride $\mathrm{PdH}_{2}$, which he assumed to crystallize in the cubic fluorite structure coming with a tetrahedral coordination of the $\mathrm{Pd}$ atoms by hydrogen. In the present structure, we alternatively considered the pyrite structure as a starting point for the structural relaxations. This was motivated by its close relationship to the fluorite structure as well as by a variety of exciting results recently obtained for pyrite-type materials $67-70$. Yet, in contrast to the fluorite structure, in the pyrite structure the metal atoms are octahedrally coordinated and the hydrogen atoms form closely coupled dimers parallel to the $\langle 111\rangle$ direction. To be specific, the pyrite structure is based on a simple cubic lattice with space group $P a \overline{3}\left(T_{h}^{6}\right)$ (see Ref. 67 and references therein). The atoms are located at the Wyckoff positions (4a) and (8c), which are special cases of the general position $(24 \mathrm{~d}): \pm(\mathrm{x}, \mathrm{y}, \mathrm{z}), \pm\left(\frac{1}{2}-\mathrm{x},-\mathrm{y}, \frac{1}{2}+\mathrm{z}\right)$, $\pm\left(-\mathrm{x}, \frac{1}{2}+\mathrm{y}, \frac{1}{2}-\mathrm{z}\right), \quad \pm\left(\frac{1}{2}+\mathrm{x}, \frac{1}{2}-\mathrm{y},-\mathrm{z}\right), \quad$ (and cyclic permutations of $\mathrm{x}, \mathrm{y}$, and $\mathrm{z}$ ) with $\mathrm{x}=\mathrm{y}=\mathrm{z}=0$ and $\mathrm{x}=\mathrm{y}=\mathrm{z} \approx 0.4$, respectively. The fluorite structure arises when the (8c) position is given by $\mathrm{x}=\mathrm{y}=\mathrm{z}=0.25$, in which special case the crystal symmetry is raised to the full cubic group $F m \overline{3} m\left(O_{h}^{5}\right)$. As for the monohydride structures, full optimizations including the lattice parameter and the hydrogen position were performed using VASP. However, the pyrite structe proved unstable inasmuch as optimization of this structure eventually led to the fluorite structure. This is most probably due to strong $\mathrm{H}-\mathrm{H}$ bonds coupling the characteristic hydrogen dimers in the pyrite structure, which undermine the $\mathrm{Pd}-\mathrm{H}$ bonding and thus lead to a positive energy of formation.

As a consequence, only the fluorite structure was included in the subsequent analysis of the electronic structure. The lattice parameter of the optimized structure was $4.37,4.39$, and $4.45 \AA$ as obtained from LDA, PBEsol, and PBE calculations, respectively. In addi- tion, the heat of formation was calculated as $-0.54 \mathrm{eV}$, $-0.22 \mathrm{eV}$, and $+0.10 \mathrm{eV}$, respectively, all per formula unit. These values should be compared to the heats of formation of $\mathrm{PdH}$ calculated from these three schemes, which amount to $-0.51 \mathrm{eV},-0.36 \mathrm{eV}$, and $-0.16 \mathrm{eV}$, respectively (the LDA value for $\mathrm{PdH}$ compares nicely with the value of $-0.53 \mathrm{eV}$ reported by Isaeva et al..$\left.^{33}\right)$. Hence, within the GGA schemes, $\mathrm{PdH}$ is more stable than $\mathrm{PdH}_{2}$, which is even on the verge of forming a thermodynamically stable compound.

The calculated (partial) densities of states arising from the subsequent ASW calculations are shown in Fig. 7. As for the monohydrides, we find dominant contribu-

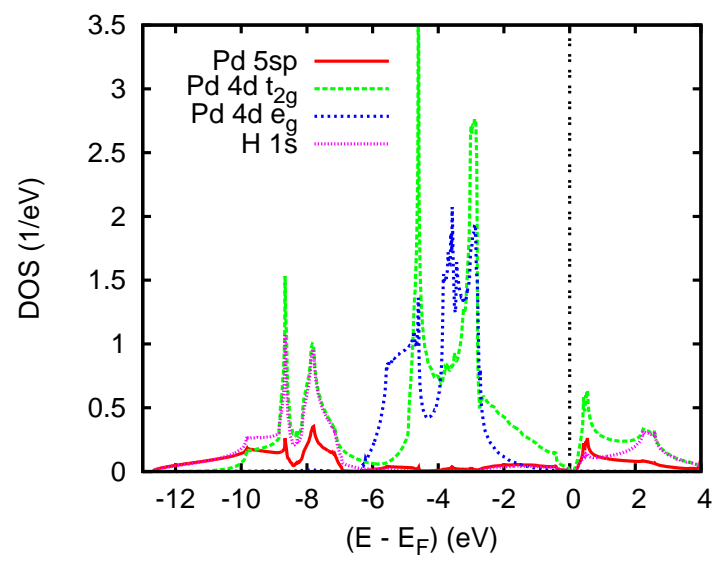

FIG. 7: Partial densities of states of $\mathrm{PdH}_{2}$ in the fluorite structure.

tions from the $\mathrm{Pd} 4 d$ states, which are complemented by small admixtures from the $5 s p$ states. In addition, considerable contributions from the $\mathrm{H} 1 s$ states are observed, especially between -12 and $-7 \mathrm{eV}$ and above the Fermi energy. Contributions from the $\mathrm{Pd} 4 d$ states in these energy ranges are of similar size as the $\mathrm{H} 1 s$ contributions and of almost identical shape, indicating the strong covalent bonding of these states. According to Fig. 7 and very similar to the situation in zincblendetype $\mathrm{PdH}$ this $\sigma$-type bonding is maintained exclusively by the $t_{2 g}$ manifold, whereas the $e_{g}$ orbitals have no overlap with the $\mathrm{H} 1 s$ states at all. Two additional points are especially noteworthy: In contrast to the partial densities of states of zincblende-type $\mathrm{PdH}$ as shown in the lower part of Fig. 2 the split-off band of $\mathrm{PdH}_{2}$ between -12 and $-7 \mathrm{eV}$ falls into two main peaks, which both carry a considerable weight of the $\mathrm{H} 1 s$ orbitals. This is due to the higher amount of electrons contributed from the hydrogen atoms. Yet, as is obvious from the similar $4 d$ and $1 s$ weights in that energy range, these electrons are not simply transferred to the metal atoms as in an ionic scenario but rather participate in the covalent bonding.

Second, as a detailed comparison of the partial densities of states of the dihydride to those of zincblende-type $\mathrm{PdH}$ reveals, the two groups of bands ranging from -12 to $-7 \mathrm{eV}$ and from $-7 \mathrm{eV}$ to the Fermi energy correspond 
to the two groups of bands displayed in the lower part of Fig. 2 for zincblende $\mathrm{PdH}$. Hence, in $\mathrm{PdH}_{2}$, we observe an additional third group of bands, which are found above $\mathrm{E}_{\mathrm{F}}$. These bands may be regarded as the antibonding $\mathrm{Pd}$ $4 d-\mathrm{H} 1 s$ states, which together with their bonding partners between -12 and $-7 \mathrm{eV}$ embrace the central group of metal $4 d$ states.

The gross picture is complemented by a closer inspection of the band structure as given in Fig. 8 which reveals

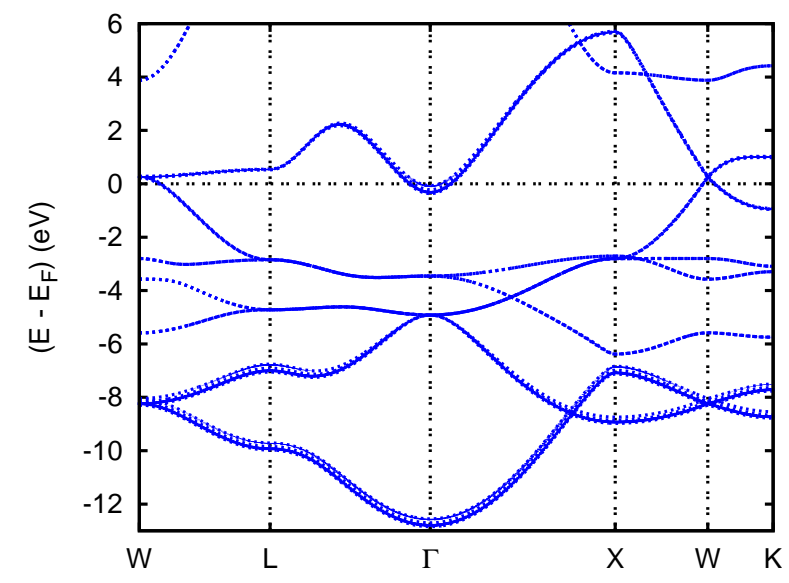

FIG. 8: Electronic bands of $\mathrm{PdH}_{2}$. Contributions of the $\mathrm{H} 1 s$ orbitals are highlighted. The width of the bars given with each band is a measure of the $\mathrm{H} 1 s$ contribution to the respective wave function.

the stronger distortion of the bands of elemental Pd as compared to $\mathrm{PdH}$ due to the larger hydrogen content of the dihydride. In particular, as mentioned above, now the two lowest bands of the Pd $4 d$ complex comprise a finite contribution of the $\mathrm{H} 1 s$ orbitals. In addition, the second band shows strong hybridization with a new single band, which starts just below the Fermi energy and extends up to about $6 \mathrm{eV}$ with the hybridization being strongest at the $\Gamma$ point. This new band leads to the above mentioned additional contribution to the density of states.

As before, we complement the previous analysis of the electronic structure and partial densities of states with an investigation of the chemical bonding properties in terms of the partial covalence energies, which are displayed in Fig. 9. Overall, the results are quite comparable to those of zincblende-type $\mathrm{PdH}$. Again, the $\mathrm{Pd} 4 d-\mathrm{H} 1 s$ overlap plays an essential role for stabilizing the dihydride, with the split-off bands between -12 and $-7 \mathrm{eV}$ comprising the bonding contributions. However, due to the downshift of the central group of $4 d$ bands, occupation of the corresponding antibonding states sets in at a lower energy. As a consequence, we observe the destabilization of dihiydride as compared to PdH. Finally, occupied antibonding $5 s p-4 d$ and $4 d-4 d$ orbitals even enhance this effect. In passing, we mention the somewhat unexpected negative $d$ - $d$ covalence energies above the Fermi energy. However, it should be taken into account that in this

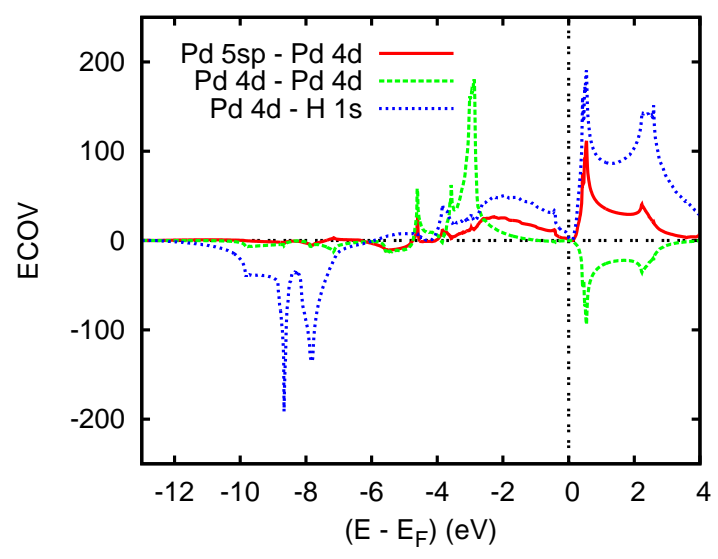

FIG. 9: Partial covalence energies of $\mathrm{PdH}_{2}$ in the fluorite structure.

energy region there is a strong similarity of the $\mathrm{Pd} 4 d$ and $5 s p$ partial densities of states as revealed by Fig. 7 , pointing to interatomic covalent $4 d-5 s p$ bonding, which according to to Fig. 9 adds to the $d$ - $d$ interactions and counterbalances these.

\section{C. $\mathrm{Pd}_{3} \mathrm{H}_{4}$ phase}

As noted in the introduction, recent experiments have shown that palladium hydride tends to form palladium vacancies at about $700-800^{\circ} \mathrm{C}$ and $5 \mathrm{GPa}$ hydrogen pressure. This induces a phase transition to a stable $\mathrm{Pd}_{3} \mathrm{H}_{4}$ composition, which forms an ordered structure arising from $\mathrm{PdH}$ on removal of e.g. the $\mathrm{Pd}$ atoms at the corners of the cubic cell 24,33 . Following Isaeva et al. we investigated $\mathrm{Pd}_{3} \mathrm{H}_{4}$ in both the so modified rocksalt and zincblende structures 33 . However, while these authors focused throughout on the thermodynamic stability of the two structures, we here concentrate on the electronic structure and the structure-property relationships.

Yet, again the investigation was initiated by structure optimizations employing the VASP code. The results as obtained from different approximations to the exchange-correlation functional are summarized in Table III As before, we find an increase of the lattice constant on going from LDA to PBEsol and PBE. Our results are in reasonable agreement with those of Isaeva et al. but deviate from the larger experimental value. However, they do reproduce the volume reduction on going from $\mathrm{PdH}$ to the vacancy phase. Finally, we find the zincblende-derived structure at much higher energies than the rocksalt-derived structure, again in agreement with experimental data.

The results of the subsequent calculations of the electronic structure using the ASW code are displayed in Fig. 10. Here, from the distinction of the $\sigma$ - and $\pi$-bonding manifolds of the $\mathrm{Pd} 4 d$ states, the strong $\sigma$-type overlap 
TABLE II: Calculated equilibrium properties of $\mathrm{Pd}_{3} \mathrm{H}_{4}$ in the rocksalt (RS) and zincblende (ZB) structures: total energies $E$ per formula unit and lattice constants $a$ within LDA/PBEsol/PBE. Experimental data (from Ref. 22) are added for comparison.

\begin{tabular}{l|l|lll|lll}
\hline \hline & & \multicolumn{2}{|c}{$E-E_{R S}(\mathrm{meV})$} & \multicolumn{3}{c}{$a(\AA)$} \\
\hline \multirow{2}{*}{ RS } & this work & & \multicolumn{2}{c}{0} & 3.88 & 3.91 & 3.96 \\
& Ref. 33 & & & & 3.92 & & \\
& Ref. 22 & & & & & 4.02 & \\
\hline ZB & this work & 447 & 406 & 254 & 3.95 & 3.98 & 4.06 \\
& Ref. 33 & & & & 4.04 & & \\
\hline \hline
\end{tabular}
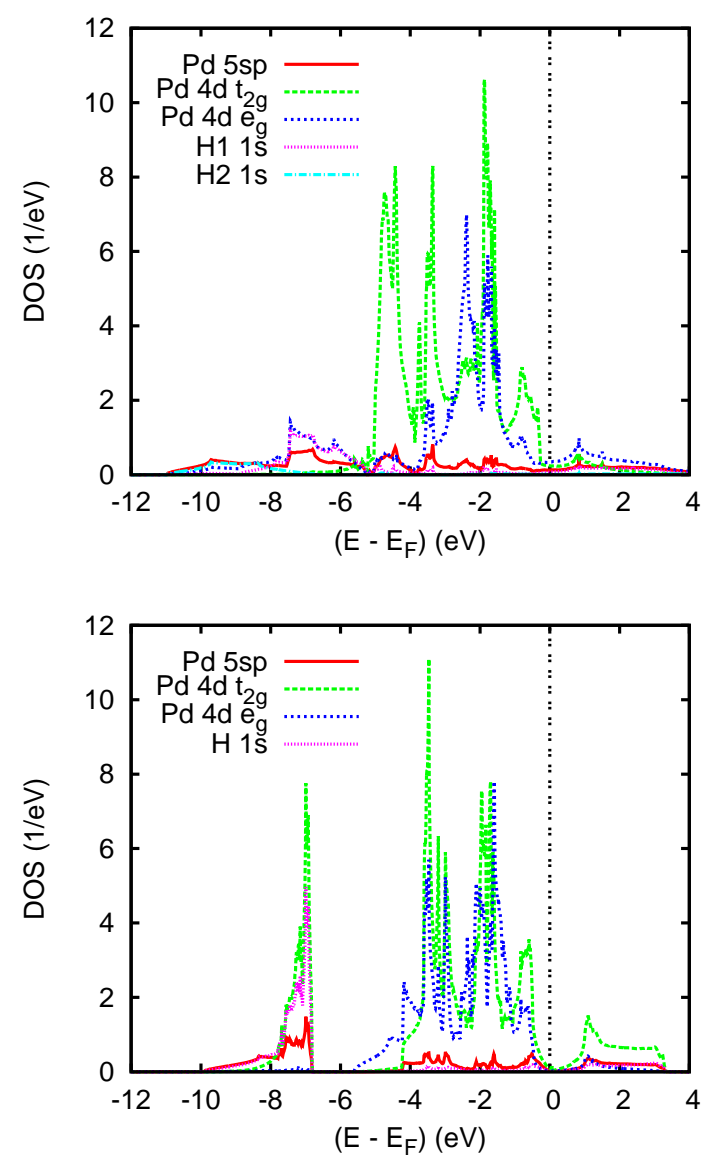

FIG. 10: Partial densities of states of $\mathrm{Pd}_{3} \mathrm{H}_{4}$ in the rocksalt ( $\mathrm{H}$ in octahedral site, top) and zincblende ( $\mathrm{H}$ in tetrahedral site, bottom) structure.

of the $\mathrm{H} 1 s$ orbitals with the $e_{g}$ and $t_{2 g}$ orbitals, respectively, in the rocksalt and zincblende structures becomes obvious. In addition, we point out that removal of the $\mathrm{Pd}$ atoms at the corners of the cube leads to two different types of hydrogen atoms in the rocksalt structure, namely, $\mathrm{H} 1$ atoms located in the middle of the edges and $\mathrm{H} 2$ at the center of the cube. In contrast, in the zincblende-derived vacancy structure, still all hydrogen atoms are equivalent by symmetry. This has interesting consequences for the electronic states. While for the zincblende-derived structure only one split-off band in the energy range from $-10 \mathrm{eV}$ to $-7 \mathrm{eV}$ is observed, the split-off band in the rocksalt structure falls into two parts: The rather weak low-energy part between $-11 \mathrm{eV}$ to $-7.5 \mathrm{eV}$ arises from overlap of the $\mathrm{Pd} 4 d$ states with the $1 s$ states of the single central H2-type atoms, whereas the strong high-energy part between $-7.5 \mathrm{eV}$ to $-5 \mathrm{eV}$ is due to the overlap with the orbitals of the three H1-type atoms. This difference in $d-s$ splitting is easily explained from the fact that the H2-type atoms at the center of the cubic cell have six Pd neighbors, whereas the H1-type atoms at the edges have only four such neighboring $\mathrm{Pd}$ atoms. As a consequence, these latter atoms experience less overlap with the $4 d$ orbitals. Finally, we mention the dip of all partial densities of states just above the Fermi energy, which for the dihydride was observed at the Fermi energy and attributed to the complete occupation of the $d$ shell and the onset of mainly $1 s$-derived bands.

Finally, partial covalence energies were calculated and could be well understood from those obtained for the monohydrides and dihydride as shown in Figs. 4 and 9 taking into account the increased H:Pd ratio as compared to $\mathrm{PdH}$ and the resulting appearance of the additional group of bands as discussed in Sec. IIIB. In particular, while strong $\mathrm{Pd} 4 d-\mathrm{H} 1 s$ bonding stabilizes the structure, antibonding $d$ - $d$ interactions tend to undermine the overall stability.

\section{SUMMARY}

In conclusion, three different types of palladium hydrides with varying hydrogen concentration have been investigated by first principles electronic structure calculations using two complementary tools, namely, the VASP and the ASW code. For the monohydride PdH the rocksalt structure has been identified as the ground state in agreement with previous experimental and theoretical work. However, were it not for the zero-point energy, the zincblende structure would be more stable. In particular, the strong difference in zero-point energy results from the larger voids available for the hydrogen atoms and consequently much softer $\mathrm{Pd}-\mathrm{H}$ bonds in the rocksalt structure as compared to the zincblende and wurtzite structure. As compared to Pd metal, insertion of hydrogen into the face-centered cubic lattice leads to the formation of bonding and antibonding $4 d-1 s$ states, which are found below and above the remaining $\mathrm{Pd} 4 d$ bands, respectively. Especially, the bonding split-off bands are easily identified and underpinned by recent XPS and UPS data. In addition, hydrogenation leads to the upshift of the Fermi level to an energy range, where the density of states is low. As a consequence, the $\mathrm{Pd}-\mathrm{H}$ bonding prevails over magnetovolume effects, leading to the suppression of magnetic order. For the dihydride $\mathrm{PdH}_{2}$, the fluorite structure is found to be more stable than the pyrite 
structure albeit with a very small difference in heat of formation. The higher hydrogen content leads to an increased complexity of the band structure as compared to the metal and the monohydride due to the formation of a second split-off band below the central $4 d$ bands and of an additional $1 s$-derived band above this group. Finally, for supervacant $\mathrm{Pd}_{3} \mathrm{H}_{4}$, our calculations have established the rocksalt-type ground state. A detailed analysis of the electronic structures established the critical importance of strong covalent bonds of directional orbitals for the thermodynamic stability of all these hydrides.
* Corresponding author abdeslam.houari@univ-bejaia.dz

† matar@icmcb-bordeaux.cnrs.fr

$\ddagger$ veyert@materialsdesign.com

1 N. L. Rosi, J. Eckert, M. Eddaoudi, D. T. Vodak, J. Kim, M. O'Keeffe, and O. M. Yaghi, Science 300, 1127 (2003).

2 I. P. Jain, C. Lal, and A. Jain, Int. J. Hydrogen Energy 35, 5133 (2010).

3 M. Felderhoff and B. Bogdanovic, Int. J. Mol. Sci. 10, 325 (2009).

${ }^{4}$ N. W. Ashcroft, Phys. Rev. Lett. 92, 187002 (2004).

5 S. F. Matar, J. Prog. Solid State Chem. 41, 55 (2013).

6 T. Graham, Proc. Roy. Soc. 17, 212 (1869).

7 F. A. Lewis, Platin. Met. Rev. 4, 132 (1960).

${ }^{8}$ F. A. Lewis, The Palladium Hydrogen System (Academic Press, New York 1967).

${ }^{9}$ W. M. Muller, J. P. Blackledge, and G. G. Libowitz, Metal Hydrides (Academic Press, New York 1968).

10 D. G. Westlake, C. B. Satterthwaite, and J. H. Weaver, Phys. Today 31(11), 32 (1978).

11 G. Alefeld and J. Völkl, eds., Hydrogen in Metals I, Topics Appl. Phys. 28 (Springer, Berlin 1978); Hydrogen in Metals II, Topics Appl. Phys. 29 (Springer, Berlin 1978).

12 F. A. Lewis, Platin. Met. Rev. 26, 20 (1982).

13 T. B. Flanagan and W. A. Oates, Annu. Rev. Mater. Sci. 21, 269 (1991).

14 Y. Fukai, The Metal Hydrogen System, Basic Bulk Properties (Springer, Berlin 1993).

15 T. B. Flanagan and Y. Sakamoto, Int. J. Hydrogen Energy 19, 151 (1994).

16 F. D. Manchester, A. San-Martin, and J. M. Pitre, J. Phase Equilib. 15, 62 (1994).

17 J. E. Worsham, J. R. M. K. Wilkinson, and C. G. Shull, J. Phys. Chem. Solids 3, 303 (1957).

18 G. A. Ferguson, A. I. Schindler, T. Tanaka, and T. Morita, Phys. Rev. 137, A483 (1965).

19 M. P. Pitt and E. M. Gray, Europhys. Lett. 64, 344 (2003).

20 A. C. Switendick, Solid State Commun. 8, 1463 (1970).

21 A. C. Switendick, Int. J. Quantum Chem. 5, 459 (1971).

${ }^{22}$ Y. Fukai and N. Okuma, Phys. Rev. Lett. 73, 1640 (1994).

23 V. F. Degtyareva, Proceedings of XI. International Conference on Hydrogen Materials Science and Chemistry of Carbon Nanomaterials, p. 202 (2009).

24 S. Harada, D. Ono, H. Sugimoto, and Y. Fukai, J. Alloys Comp. 446, 474 (2007).

${ }^{25}$ C. D. Gelatt, Jr., H. Ehrenreich, and J. A. Weiss, Phys. Rev. B 17, 1940 (1978).

${ }^{26}$ M. Gupta and A. J. Freeman, Phys. Rev. B 17, 3029 (1978).

27 A. R. Williams, J. Kübler, and C. D. Gelatt, Jr., Phys. Rev. B 19, 6094 (1979).

28 M. Methfessel and J. Kübler, J. Phys. F: Met. Phys. 12, 141 (1982).

29 C. T. Chan and S. G. Louie, Phys. Rev. B 27, 3325 (1983).
30 C. Elsässer, M. Fähnle, K. M. Ho, and C. T. Chan, Physica B 172, 217 (1991).

31 R. Caputo and A. Alavi, Mol. Phys. 101, 1781 (2003).

32 M. S. Dyer, PhD-thesis, University of Cambridge, 2006.

${ }^{33}$ L. E. Isaeva, D. I. Bazhanov, E. E. Isaev, S. V. Eremeev, S. E. Kulkova, and I. A. Abrikosov, Int. J. Hydrogen Energy 36, 1254 (2011).

34 S. F. Matar, B. Chevalier, V. Eyert, and J. Etourneau, Solid State Sciences 5, 1385 (2003).

35 S. F. Matar, J. Prog. Solid State Chem. 38, 1 (2010).

36 P. Hohenberg and W. Kohn, Phys. Rev. 136, B864 (1964).

37 W. Kohn and L. J. Sham, Phys. Rev. 140, A1133 (1965).

38 G. Kresse and J. Furthmüller, Phys. Rev. B 54, 11169 (1996); Comput. Mater. Sci. 6, 15 (1996).

39 MedeA $^{\circledR}$ 2.14, Materials Design, Inc. (2014).

40 J. P. Perdew and A. Zunger, Phys. Rev. B 23, 5048 (1981).

41 J. P. Perdew, K. Burke, and M. Ernzerhof, Phys. Rev. Lett. 77, 3865 (1996).

42 J. P. Perdew, A. Ruzsinszky, G. I. Csonka, O. A. Vydrov, G. E. Scuseria, L. A. Constantin, X. Zhou, and K. Burke, Phys. Rev. Lett. 100, 136406 (2008); A. E. Mattsson, R. Armiento, and T. R. Mattsson, Phys. Rev. Lett. 101, 239701 (2008); J. P. Perdew, A. Ruzsinszky, G. I. Csonka, O. A. Vydrov, G. E. Scuseria, L. A. Constantin, X. Zhou, and K. Burke, Phys. Rev. Lett. 101, 239702 (2008); J. P. Perdew, A. Ruzsinszky, G. I. Csonka, O. A. Vydrov, G. E. Scuseria, L. A. Constantin, X. Zhou, and K. Burke, Phys. Rev. Lett. 102, 039902(E) (2009).

43 P. E. Blöchl, Phys. Rev. B 50, 17953 (1994).

44 G. Kresse and D. Joubert, Phys. Rev. B 59, 1758 (1999).

${ }^{45}$ H. J. Monkhorst and J. D. Pack, Phys. Rev. B 13, 5188 (1976).

${ }^{46}$ K. Parlinski, Z. Q. Li, and Y. Kawazoe, Phys. Rev. Lett. 78, 4063 (1997).

47 V. Eyert, Int. J. Quantum Chem. 77, 1007 (2000)

48 V. Eyert, The Augmented Spherical Wave Method, Lect. Notes Phys. 849 (Springer, Berlin Heidelberg 2013).

49 Z. Wu and R. E. Cohen, Phys. Rev. B 73, 235116 (2006).

50 V. Eyert, and K.-H. Höck, Phys. Rev. B 57, 12727 (1998).

51 V. Eyert, J. Comput. Phys. 124, 271 (1996).

52 P. E. Blöchl, O. Jepsen, and O. K. Andersen, Phys. Rev. B 49, 16223 (1994).

53 M. S. Methfessel, Phys. Rev. B 38, 1537 (1988).

${ }^{54} \mathrm{C}$. Kittel, Introduction to solid state physics (Wiley, New York, Chichester 1996).

55 J. E. Schirber and B. Morosin, Phys. Rev. B 12, 117 (1975)

56 A. C. Switendick, J. Less Common Metals 130, 249 (1987).

57 E. Wimmer, W. Wolf, J. Sticht, P. Saxe, C. B. Geller, R. Najafabadi, and G. A. Young, Phys. Rev. B 77, 134305 (2008).

58 L. Schlapbach and J. P. Burger, J. Phys. Lett. 43, L273 (1982). 
59 P. A. Bennett and J. C. Fuggle, Phys. Rev. B 26, 6030 (1982).

60 S. Sinha, S. Badrinarayanan, and A. P. B. Sinha, J. Phys. F: Met. Phys. 16, L229 (1986).

61 S. F. Matar, Comput. Mat. Sci. 69, 424 (2013).

${ }^{62}$ H. Kohlmann, N. Kurtzemann, R. Weihrich, and T. Hansen, Z. Anorg. Allg. Chemie 635, 2399 (2009).

63 R. Hoffmann, Solids and Surfaces: A Chemists View of Bonding in Extended Structures (VCH Verlagsgesellschaft, New York 1988).

64 N. Börnsen, B. Meyer, O. Grotheer, and M. Fähnle, J. Phys.: Cond. Matt. 11, L287 (1999).
${ }^{65}$ M. Castro, Phys. Rev. B 43, 14378 (1991).

66 S. C. Hong and J. I. Lee, J. Korean Phys. Soc. 52, 1099 (2008).

67 V. Eyert, K.-H. Höck, S. Fiechter, and H. Tributsch, Phys. Rev. B 57, 6350 (1998).

${ }^{68}$ R. Weihrich, S. F. Matar, E. Betranhandy, and V. Eyert, Solid State Sciences 5, 701 (2003).

69 R. Weihrich, V. Eyert, and S. F. Matar, Chem. Phys. Lett. 373, 636 (2003).

70 A. Houari, S. F. Matar, and V. Eyert, Phys. Rev. B 82, 241201 (2010). 